\title{
Оптические свойства халькогенидных сплавов на основе теллура в дальнем инфракрасном диапазоне $(\lambda>30$ мкм)
}

\author{
(C) В.А. Рыжов, Б.Т. Мелех \\ Физико-технический институт им. А.Ф. Иоффре Российской академии наук, \\ 194021 Санкт-Петербург, Россия \\ E-mail: v.ryzhov@mail.ioffe.ru
}

(Получена 17 апреля 2017 г. Принята к печати 26 июня 2017 г.)

\begin{abstract}
Синтезированы тройные теллуридные сплавы систем $\mathrm{Ge}-\mathrm{Se}(\mathrm{Sb})-\mathrm{Te}$ и $\mathrm{Si}-\mathrm{Ge}(\mathrm{Ga})-\mathrm{Te}$ в стеклообразном и кристаллическом состояниях для использования в терагерцовом диапазоне частот. Измерены и исследованы спектры пропускания полученных сплавов в широком интервале длин волн от 0.75 до 300 мкм. Обсуждаются возможные механизмы их формирования. Сравнительный анализ результатов показывает, что наиболее перспективным является сплав $\mathrm{Ge}_{14} \mathrm{Sb}_{28} \mathrm{Te}_{56}$ системы GST, фононный спектр которого лежит в диапазоне $40-280 \mathrm{~cm}^{-1}$, ограничивая длинноволновое окно прозрачности этого сплава 35 мкм. Оптимизация состава $\mathrm{Ge}_{14} \mathrm{Sb}_{28} \mathrm{Te}_{56}$, удаление примесей и термообработка будут способствовать дальнейшему снижению коэффициента поглощения в дальнем инфракрасном спектре этого сплава.
\end{abstract}

DOI: 10.21883/FTP.2018.02.45446.8611

\section{1. Введение}

Одним из основных достижений науки последних трех десятилетий является освоение терагерцового (ТГц) диапазона электромагнитного спектра ( $\lambda=30-1000$ мкм). То, что раньше называли субмиллиметровым „провалом“ на шкале электромагнитных волн, теперь широко используется молекулярной спектроскопией для изучения молекулярных структур и динамики кристаллов и стекол [1].

Достигнутый прогресс в технологии для малоизученного диапазона частот привлек внимание к разработке новых ТГц-материалов. Так, для проекта Darwin были впервые использованы халькогенидные стекла на основе $\mathrm{As}, \mathrm{S}$ и $\mathrm{Se}$, позволяющие работать в окнах прозрачности атмосферы при 3-5 и 8-12 мкм [2]. Активное применение инфракрасных (ИК) волоконных световодов для оптической связи, медицины и экологии далее показало, что именно халькогенидные сплавы (XC) являются наиболее перспективным материалом, работающим с ИК излучением. Для волоконной оптики были синтезированы XC: As-S, As-Se, As-S-Se, Ge-S, $\mathrm{Ge}-\mathrm{Se}, \mathrm{Ge}-\mathrm{As}-\mathrm{S}$, As-Se-Te, Ge-As-Se, Ge-Se-Te, $\mathrm{Ga}-\mathrm{La}-\mathrm{S}$ и $\mathrm{Ge}(\mathrm{Ga})-\mathrm{Sb}-\mathrm{S}$ систем [3-5]. Устойчивый интерес к технологии получения и исследованию физико-химических свойств ХC сохраняется до сих пор. В недавних работах [6-8], выполненных в ФТИ им. А.Ф. Иоффе, были измерены оптические, акустические и фотоупругие параметры тройных и даже четверных сплавов: германий-селен-теллур ( $\mathrm{Ge}-\mathrm{Se}-\mathrm{Te})$, германий-селен-сера-теллур (Ge-Se-S-Te) и германий-кремний $(\mathrm{Si}-\mathrm{Te})$ для приборов и устройств акустооптики. Эти исследования показали, что окно прозрачности ХC, содержащих теллур, может перекрывать практически весь средний ИК диапазон в зависимости от концентрации $\mathrm{Ge}, \mathrm{Se}$ или $\mathrm{S}$ в сплаве.
Поиск ХC в качестве материала, прозрачного для длинноволнового ИК излучения активно продолжается. Возможность в результате подбора химического состава и специальных технологий синтеза изменять спектральные характеристики халькогенидных сплавов в сочетании с их способностью к формовке и вытяжке обеспечивают успех такого поиска. В этом смысле наиболее перспективными из них в настоящее время являются халькогенидные сплавы, содержащие теллур.

Задачей данной работы было создание и изучение новых сплавов систем $\mathrm{Ge}-\mathrm{Se}(\mathrm{Sb})-\mathrm{Te}$ и $\mathrm{Si}-\mathrm{Ge}(\mathrm{Ga})-\mathrm{Te}$ с целью продвижения фононного края поглощения в этих системах в терагерцовую область ИК спектра.

Необходимым условием эффективности исследований было в первую очередь знание о спектральном положении решеточных колебаний всех компонентов изученных нами сплавов. В области длин волн $\lambda>20$ мкм такие данные стали доступными лишь в последнее время благодаря успехам метода терагерцовой спектроскопии.

\section{2. Образцы и методика эксперимента}

Изготовление образцов осуществлялось с использованием исходных материалов ( $\mathrm{Ge}, \mathrm{Se}, \mathrm{Sb}, \mathrm{Te})$ марки ОСЧ. Синтез проводился в откачанных запаянных кварцевых ампулах с удлиненным конусным концом [7]. Синтез происходил при температуре $850-900^{\circ} \mathrm{C}$ в течение 5-6 ч. В процессе синтеза для гомогенизации расплава осуществлялось его многократное перемешивание. Охлаждение расплавов проводилось в конусной части ампулы в режиме остывания на „воздухе“ (скорость охлаждения 50-70 K/мин). Масса навески составляла 10 г. Это позволило оценить стеклообразующую способность расплава по величине диаметра $(D)$ сечения конуса. Стеклообразное состояние идентифицировалось по характерному раковистому излому, отсутствию ли- 
ний на дебаеграммах [7] и повышенной по сравнению с кристаллическим состоянием величине удельного электросопротивления.

Были синтезированы образцы следующих составов: $\mathrm{Ge}_{33} \mathrm{Se}_{33} \mathrm{Te}_{33}, \mathrm{Ge}_{19} \mathrm{Se}_{9} \mathrm{Te}_{72}, \mathrm{Si}_{20} \mathrm{Te}_{80}, \mathrm{Si}_{10} \mathrm{Ge}_{10} \mathrm{Te}_{80}$, $\mathrm{Si}_{15} \mathrm{Sn}_{5} \mathrm{Te}_{80}, \mathrm{Si}_{15} \mathrm{Ga}_{15} \mathrm{Te}_{70}, \mathrm{Ge}_{20} \mathrm{Se}_{70} \mathrm{Te}_{10}$ и $\mathrm{Ge}_{14} \mathrm{Sb}_{28} \mathrm{Te}_{56}$. Сплав $\mathrm{Ge}_{14} \mathrm{Sb}_{28} \mathrm{Te}_{56}$ получен как в режиме закалки на „воздухе“, так и при охлаждении расплава со скоростью 10 град/ч. Рентгенофазовый анализ показал, что основная фаза в них одинакова и близка к $\mathrm{Ge}_{0.95} \mathrm{Sb}_{2.01} \mathrm{Te}_{4}$ с параметрами гексагональной ячейки: $a=4.23 \AA$ и $c=41.1 \AA$. В закаленном образце возможна примесь эвтектики $(\mathrm{Sb}, \mathrm{Te})_{\beta}$.

Составы образцов приведены в соответствии с концентрацией исходных материалов в шихте.

Регистрация спектров пропускания в диапазоне длин волн от $\lambda=0.75$ до 2.5 мкм выполнялась с использованием спектрофотометра ИКС-14А фирмы „ЛОМО“. В диапазоне длин волн $\lambda=2.5-25$ мкм использовался спектрофотометр М-577 фирмы „Perkin-Elmer". Длинноволновые ИК спектры в области $\lambda=25-75$ мкм были зарегистрированы на спектрометре FIS-21фирмы „Hitachi“. Регистрация спектров в области $\lambda=75-300$ мкм проводилась на спектрометре, разработанном в ЛГУ и модернизированном приемником ОАП-7 и новой системой фильтрации.

Разрешение при отношении сигнала к шуму порядка 100 составляло $1-2 \mathrm{~cm}^{-1}$. Точность определения максимума полосы поглощения была $2-3 \mathrm{~cm}^{-1}$.

Образцы для записи спектров изготавливались в виде таблеток из весовой смеси 1 : 100 порошкообразного образца с полиэтиленом спектральной чистоты. Таблетки получали прессованием в атмосфере азота во избежание окисления. Все спектры были сняты при комнатной температуре. Спектр поглощения полиэтилена из них вычитался при регистрации автоматически.

\section{3. Результаты и их обсуждение}

На рис. 1 представлены типичные спектры пропускания в широком интервале длин волн $(\lambda=0.75-300$ мкм $)$ исследованных халькогенидных сплавов. Видно, что халькогенидные сплавы на основе теллура действительно имеют рекордно широкое окно прозрачности в среднем и дальнем ИК диапазонах [9]. В то же время это окно явно не зависит от концентрации теллура в сплаве (см. спектры 1 и 2). Положение коротковолнового края окна прозрачности слабо зависит от концентрации и даже состава исследованных ХС и, а его длинноволновый край содержит полосы поглощения, расположенные на одних и тех же частотах, независимо от состава и концентрации компонентов сплава. Такая картина хорошо иллюстрирует всю сложность поиска наиболее совершенного по своим спектральным характеристикам ХС для работы в дальнем ИК диапазоне.

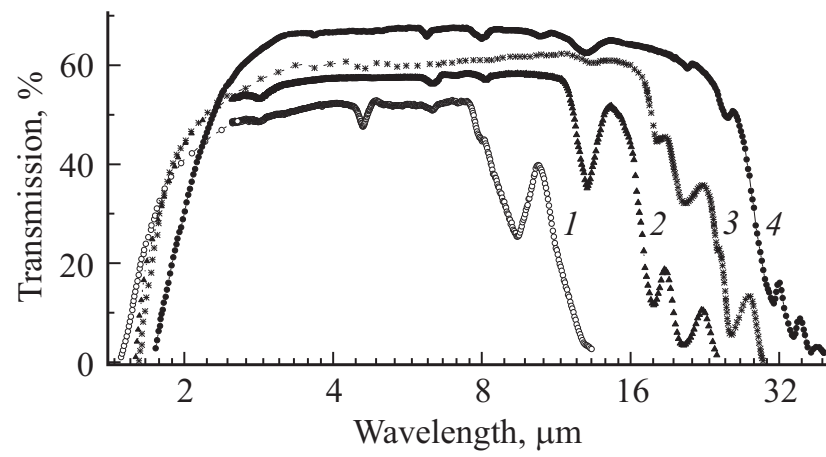

Рис. 1. Сравнение наиболее типичных спектров пропускания, изученных в этой работе сплавов: $1-\mathrm{Ga}_{15} \mathrm{Si}_{15} \mathrm{Te}_{70}$, $2-\mathrm{Ge}_{20} \mathrm{Se}_{70} \mathrm{Te}_{10}, 3-\mathrm{Ge}_{33} \mathrm{Se}_{33} \mathrm{Te}_{33}, 4-\mathrm{Ge}_{14} \mathrm{Sb}_{28} \mathrm{Te}_{56}$.

Малоинтенсивные полосы поглощения в среднем ИК диапазоне, как теперь установлено, обусловлены непременным присутствием примесей в синтезированном материале ХC [10]. Полосы поглощения при 2.3 и 2.9 мкм относят к проявлению колебательных мод гидроксильных О-Н групп, связанных с водородом и/или с атомами структурной сетки халькогенида. Полосы на длинах волн 4.3 и 6.3 мкм свидетельствуют о присутствии в образце адсорбированных молекул $\mathrm{CO}_{2}$ и $\mathrm{H}_{2} \mathrm{O}$ соответственно. Причина появления этих полос поглощения в спектре ХС объясняется присутствием паров $\mathrm{H}_{2} \mathrm{O}$ и $\mathrm{CO}_{2}$, адсорбированных образцом во время его приготовления (дробления и прессовки), а также в процессе регистрации спектров [11]. Другим классом примесей, чьи полосы поглощения присутствуют в спектрах изученных нами ХC, являются оксиды. Полосы поглощения, обусловленные колебанием атомов кислорода в паре с базовыми элементами ХC, такими как $\mathrm{Si}, \mathrm{Ge}$, $\mathrm{Se}$ и Te, расположены при 9.5, 13, 17.5 и 20 мкм соответственно. Спектральное положение полос поглощения, обусловленное присутствием примесей в изученных ХC, представлено в табл. 1. Некоторые колебательные полосы, представляющие основные химические связи между атомами в изученных ХC, также приведены в табл. 1. Именно эти полосы поглощения, как следует из дальнейшего обсуждения результатов, в идеале, когда материал очищен от примесей, определяют так называемую мультифононную границу прозрачности ХС.

Как известно, прозрачность ХС для инфракрасного излучения определяется двумя видами механизмов поглощения. Поглощение с коротковолновой стороны спектра связано с переходами электронов из валентной зоны в зону проводимости, и ширина запрещенной зоны $E_{g}$ определяет энергию перехода электронов в квазисвободное состояние. Величина $E_{g}$ коррелирует с длиной волны, определяя край оптического поглощения через соотношение: $E_{g}=h c / \lambda g$, где $E_{g}$ в эВ, $h-$ постоянная Планка в эВ·c, $c$ - скорость света в $\left(\right.$ мкм $\left.\cdot \mathrm{c}^{-1}\right)$ и $\lambda_{g}$ - длина волны в мкм. 
Таблица 1. Положение характеристических пиков в ИК спектрах ХС

\begin{tabular}{|c|c|c|c|c|}
\hline № & Образец & $\begin{array}{c}\text { Положение пиков, } \\
\mathrm{cm}^{-1} \text { (мкм) }\end{array}$ & $\begin{array}{c}\text { Интенсивности } \\
\text { пиков }\end{array}$ & $\begin{array}{l}\text { Отнесение } \\
\text { пиков }\end{array}$ \\
\hline 1 & $\mathrm{Ge}_{33} \mathrm{Se}_{33} \mathrm{Te}_{33}$ & $\begin{array}{c}3517(2.85) \\
1567(6.3) \\
766(13.0) \\
562(17.7) \\
494(20.0) \\
390(25.7) ; 405(25.6) \\
255(39.0)\end{array}$ & $\begin{array}{c}\text { Очень слабый } \\
\text { Очень слабый } \\
\text { Сильный } \\
\text { Сильный } \\
\text { Очень сильный } \\
\text { Очень сильный } \\
\text { Очень сильный }\end{array}$ & $\begin{array}{c}\mathrm{H}_{2} \mathrm{O} \\
\mathrm{H}_{2} \mathrm{O} \\
\mathrm{Ge}-\mathrm{O} ; \mathrm{Se}-\mathrm{O} ; \mathrm{Te}-\mathrm{O} \\
\text { 3-phonon } \mathrm{Se}-\mathrm{Te} ; \mathrm{Ge}-\mathrm{Te} \\
\text { 2-phonon } \mathrm{Ge}-\mathrm{Se} \\
\text { 2-phonon } \mathrm{Se}-\mathrm{Te} ; \mathrm{Ge}-\mathrm{Te} \\
\mathrm{Ge}-\mathrm{Se}\end{array}$ \\
\hline 2 & $\mathrm{Ge}_{19} \mathrm{Se}_{70} \mathrm{Te}_{10}$ & $\begin{array}{c}1300(7.7) \\
820(13.3) \\
<550(>18.5)\end{array}$ & $\begin{array}{l}\text { Очень слабый } \\
\text { Очень сильный } \\
\text { Очень сильный }\end{array}$ & $\begin{array}{c}\mathrm{Ge}-\mathrm{O} \\
\mathrm{Ge}-\mathrm{O} ; \mathrm{Se}-\mathrm{O} \\
\text { 2- phonon } \mathrm{Ge}-\mathrm{Se}\end{array}$ \\
\hline 3 & $\mathrm{Si}_{20} \mathrm{Te}_{80}$ & $\begin{array}{c}2180(4.6) \\
1800(5.5) ; 1700(5.9) \\
1050(9.5) \\
740(14.0)\end{array}$ & $\begin{array}{c}\text { Слабый } \\
\text { Очень слабый } \\
\text { Очень сильный } \\
\text { Очень сильный }\end{array}$ & $\begin{array}{l}\mathrm{CO}_{2} \\
\mathrm{H}_{2} \mathrm{O} \\
\mathrm{Si}-\mathrm{O} \\
\mathrm{Te}-\mathrm{O}\end{array}$ \\
\hline 4 & $\mathrm{Si}_{15} \mathrm{Ga}_{15} \mathrm{Te}_{70}$ & $\begin{array}{l}3500(2.85) \\
2400(4.2) \\
2150(4.65) \\
1040(9.5) \\
700(14.25)\end{array}$ & $\begin{array}{c}\text { Очень слабый } \\
\text { Слабый } \\
\text { Слабый } \\
\text { Сильный } \\
\text { Очень сильный }\end{array}$ & $\begin{array}{c}\mathrm{H}_{2} \mathrm{O} \\
\mathrm{CO}_{2} \\
\mathrm{CO}_{2} \\
\mathrm{Si}-\mathrm{O} \\
\mathrm{Te}-\mathrm{O} ; \mathrm{Ga}-\mathrm{O}\end{array}$ \\
\hline 5 & $\mathrm{Ge}_{14} \mathrm{Sb}_{28} \mathrm{Te}_{56}$ & $\begin{array}{l}3450(2.9) \\
2140(4.7) \\
1620(4.7) \\
1250(8.0) \\
950(10.5) \\
770(13.0) \\
490(20.6) \\
400(25.0) \\
320(31.0)\end{array}$ & $\begin{array}{c}\text { Очень слабый } \\
\text { Слабый } \\
\text { Слабый } \\
\text { Очень сильный } \\
\text { Очень сильный } \\
\text { Очень сильный } \\
\text { Плечо } \\
\text { Плечо } \\
\text { Плечо }\end{array}$ & $\begin{array}{c}\mathrm{H}_{2} \mathrm{O} \\
\mathrm{CO}_{2} \\
\mathrm{H}_{2} \mathrm{O} \\
\mathrm{Ge}-\mathrm{O} \\
\mathrm{Sb}-\mathrm{O}-\mathrm{Sb} \\
\mathrm{Te}-\mathrm{O} \\
\text { 3-phonon } \mathrm{Sb}-\mathrm{Te} \\
\text { 2-phonon } \mathrm{Ge}-\mathrm{Te} \\
\text { 2-phonon } \mathrm{Sb}-\mathrm{Te}\end{array}$ \\
\hline
\end{tabular}

Ширина запрещенной зоны в ХС, подобных изученным в этой работе, в зависимости от состава изменяется от 0.5 до 1.8 эВ, т.е. их коротковолновая граница прозрачности лежит в интервале 0.75-2.5 мкм [12]. Для $\mathrm{XC}$, представленных на рис. 1, этот интервал еще уже: 0.9-1.4 мкм. Он практически не зависит от состава ХC, обогащенного теллуром. В отличие от длинноволнового края окна прозрачности, который для сплава состава $\mathrm{Ge}_{15} \mathrm{Si}_{15} \mathrm{Te}_{70}$ лежит при $\sim 13$ мкм, а для сплава состава $\mathrm{Ge}_{14} \mathrm{Sb}_{28} \mathrm{Te}_{56}$ при $\sim 32$ мкм. Длинноволновый край окна прозрачности определяется другим механизмом: мультифононным поглощением. Это поглощение обусловлено взаимодействием излучения с колебательными модами атомов материала. В упрощенной двухатомной модели энергия фонона $\left(E_{f}\right)$ коррелирует с силовой постоянной связи $(k)$ и приведенной массой $m\left(1 / m=1 / m_{1}+1 / m_{2}\right)$ колеблющихся атомов через следующее соотношение: $E_{f}=h c v_{f}$, где $v_{f}=(2 \pi)^{-1} \cdot(k / m)^{1 / 2}$. Из этих уравнений следует, что энергия фононов обратно пропорциональна массе атомов, составляющих сплав. Таким образом, наличие в составе ХC тяжелых элементов смещает край фононного поглощения к более низким частотам. Поэтому сплавы, содержащие теллур, являются наилучшим кандидатом для использования их в терагерцовом диапазоне.

Однако, из-за плохой стеклообразующей способности теллура с его металлоподобными свойствами и тенденцией к кристаллизации такие сплавы должны содержать добавки. Чаще всего это более легкие элементы типа германия, селена, кремния, олова и тому подобные с дефицитом электронов для локализации свободных электронов в сплаве. Такие добавки могут существенно сузить окно прозрачности. Поэтому практика создания и использования теллуридного стекла - это дело подбора и оптимизации его состава.

Не последнюю роль и необходимым условием эффективного поиска прозрачных для ИК излучения материалов является знание о спектральном положении фононного поглощения всех его компонентов. В области длин волн $\lambda>20$ мкм такие данные стали доступными лишь в последнее время благодаря успешному освоению ТГц-диапазона частот.

На рис. 2 представлены фононные спектры решеточных колебаний $\mathrm{Si}, \mathrm{Ge}, \mathrm{Se}$, а также $\mathrm{Sb}$. Видно, что 

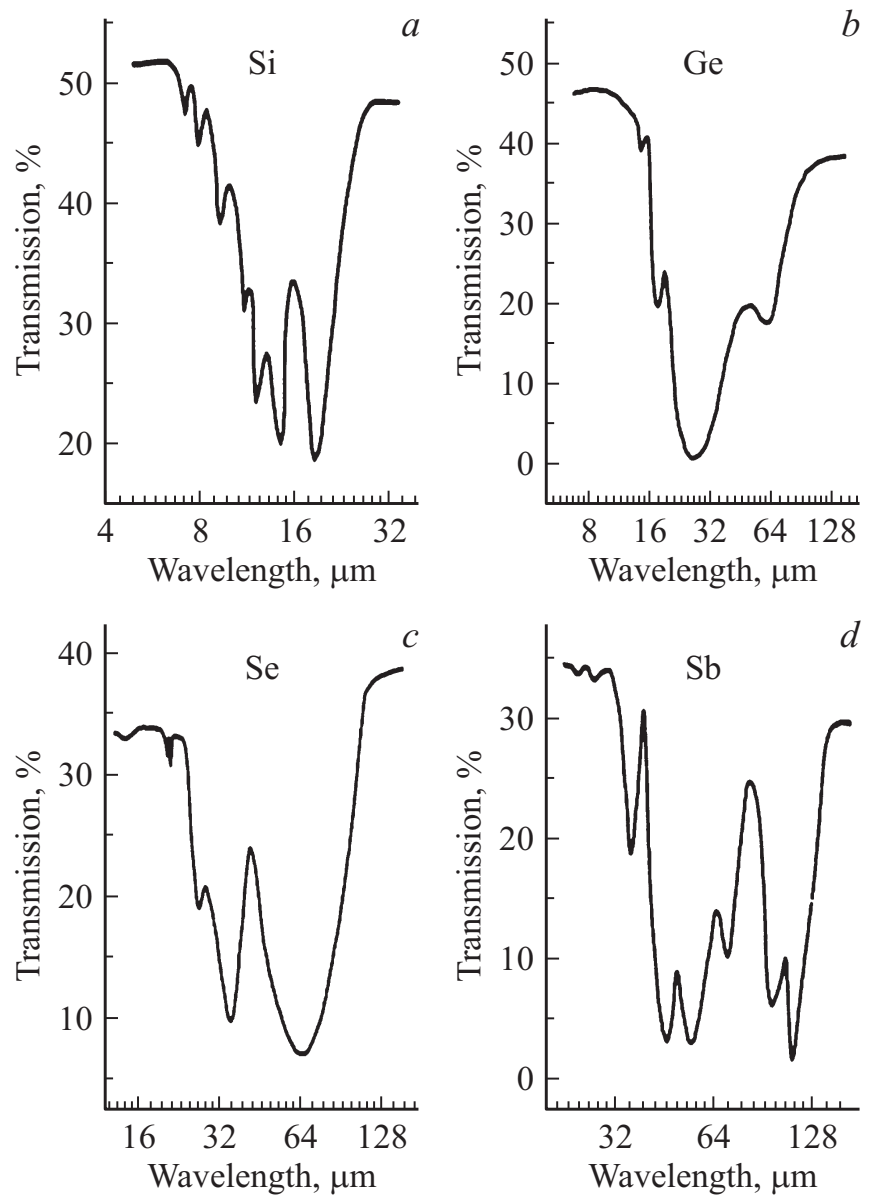

Рис. 2. Фононные спектры решеточных колебаний $\mathrm{Si}(a)$, $\mathrm{Ge}(b), \mathrm{Se}(c)$ и $\mathrm{Sb}(d)$.

теллуридные сплавы, содержащие в качестве добавки $\mathrm{Si}$, будут иметь более узкое окно прозрачности в средней ИК области (до $\sim 8$ мкм), чем в случае добавки $\mathrm{Sb}$, спектр фононного поглощения которого смещен к 32 мкм. Кроме того видно, что спектры фононного поглощения $\mathrm{Si}, \mathrm{Ge}, \mathrm{Se}$ и $\mathrm{Sb}$ занимают ограниченный диапазон длин волн. За его пределами такие материалы снова прозрачны вплоть до радиочастот [13]. Таким образом, у теллуридного сплава, содержащего в качестве добавки кремний, это „второе“ окно прозрачности в ТГц-области оказывается больше.

Разумеется, поскольку для улучшения механических, тепловых и оптических свойств ХС в его состав, как правило, вводится несколько разнородных химических элементов, спектральное положение фононного поглощения такой системы определяется не только суперпозицией фононных спектров ее составляющих. Фононный край окна прозрачности в этом случае зависит также от структуры ХС и взаимодействий между его компонентами.

Для получения такой информации были проанализированы спектры фононного поглощения вновь созданных сплавов систем $\mathrm{Ge}-\mathrm{Se}(\mathrm{Sb})-\mathrm{Te}$ и $\mathrm{Si}-\mathrm{Ge}(\mathrm{Ga})-\mathrm{Te}$.
Из рис. 3 видно, что длинноволновый край окна прозрачности ХC, представленных на рис. 1, действительно определяется положением спектров фононного поглощения этих сплавов, при этом положение самого края $\left(\lambda_{f}=1 / v_{f}\right)$ задает наиболее легкий элемент сплава. Для приведенных масс пар атомов выполняется условие $m_{\mathrm{Ge}-\mathrm{Si}}<m_{\mathrm{Ge}-\mathrm{Se}}<m_{\mathrm{Ge}-\mathrm{Sb}}$ и, соответственно, для представленных на рис. $1 \mathrm{XC}, \lambda_{f} \sim 13$ мкм для $\mathrm{Ge}_{15} \mathrm{Si}_{15} \mathrm{Te}_{70}, \lambda_{f} \sim 23$ мкм для $\mathrm{Ge}_{20} \mathrm{Se}_{70} \mathrm{Te}_{10}, \lambda_{f} \sim 29$ мкм для $\mathrm{Ge}_{33} \mathrm{Se}_{33} \mathrm{Te}_{33}$ и $\lambda_{f} \sim 35$ мкм для $\mathrm{Ge}_{14} \mathrm{Sb}_{28} \mathrm{Te}_{56}$.

Чтобы оценить влияние процентного содержания элементов сплава на положение длинноволнового (мультифононного) края окна прозрачности, необходимо располагать данными об интенсивности и отнесении полос поглощения в его фононном спектре. В случае $\mathrm{Ge}_{14} \mathrm{Sb}_{28} \mathrm{Te}_{56}$, имеющего самое широкое из полученных нами сплавов окно прозрачности в ИК диапазоне, отнесение полос проводилось на основании расчета и литературных сведений о структуре подобных псевдобинарных $(\mathrm{GeTe})_{n}\left(\mathrm{Sb}_{2} \mathrm{Te}_{3}\right)_{m}$ систем, известных как GST. Состав таких систем может быть представлен в виде двух ковалентно построенных структурных единиц областей ближнего порядка - тетраэдров $\mathrm{GeTe}_{4}$ и тригональных
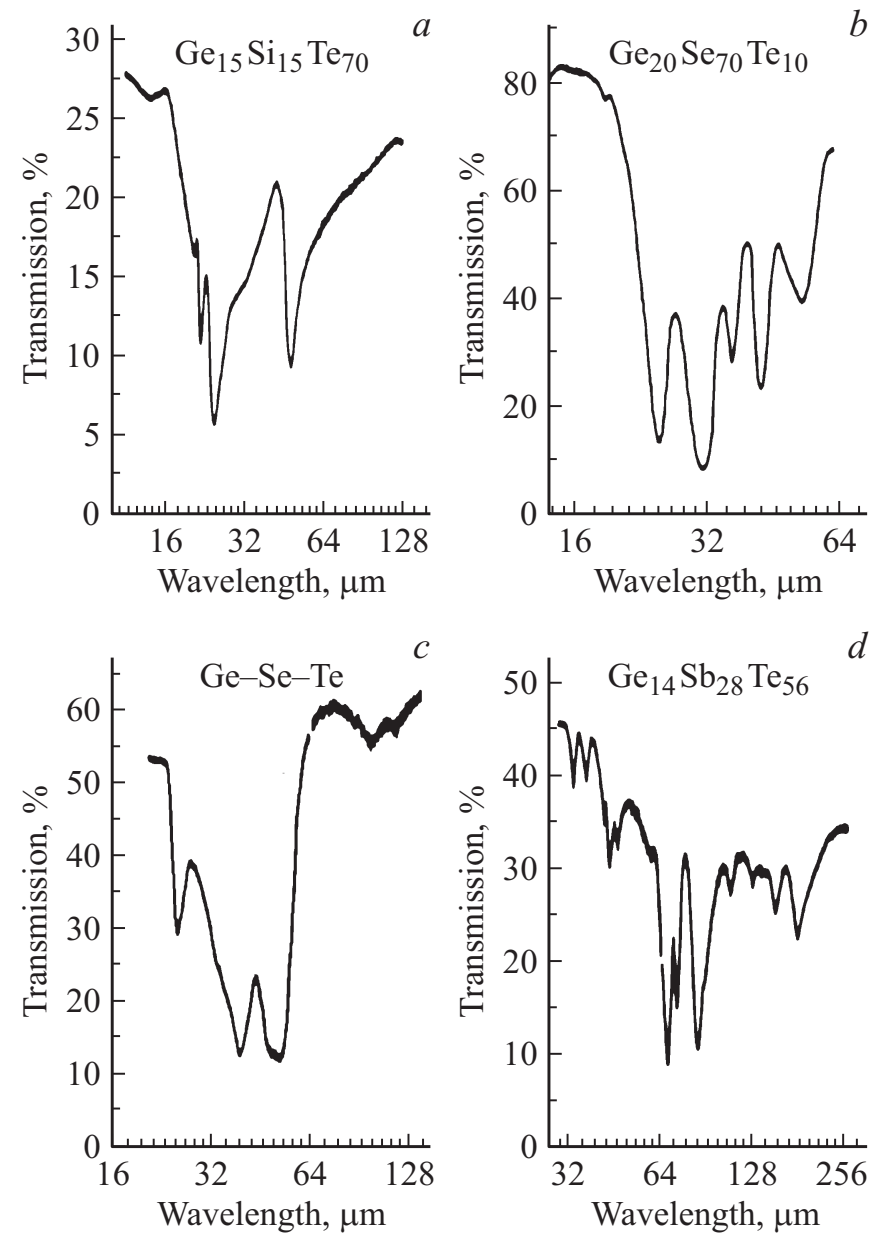

Рис. 3. Спектры фононного поглощения $\mathrm{Ge}_{15} \mathrm{Si}_{15} \mathrm{Te}_{70}(a)$, $\mathrm{Ge}_{20} \mathrm{Se}_{70} \mathrm{Te}_{10}(b), \mathrm{Ge}_{33} \mathrm{Se}_{33} \mathrm{Te}_{33}(c)$ и $\mathrm{Ge}_{14} \mathrm{Sb}_{28} \mathrm{Te}_{56}(d)$. 
Таблица 2. Частоты нормальных колебаний $\mathrm{GeTe}_{4}$ и $\mathrm{SbTe}_{3}$ в фононных спектрах $\mathrm{Ge}_{15} \mathrm{Sb}_{15} \mathrm{Te}_{70} \mathrm{u} \mathrm{Ge}_{14} \mathrm{Sb}_{28} \mathrm{Te}_{56}$

\begin{tabular}{|c|c|c|c|c|c|c|c|c|c|c|}
\hline \multirow{3}{*}{ Образец } & \multicolumn{10}{|c|}{ Частоты нормальных колебаний см${ }^{-1 *}$} \\
\hline & \multicolumn{2}{|c|}{$v 1\left(A_{1}\right)$} & \multicolumn{2}{|c|}{$v 2(E)$} & \multicolumn{2}{|c|}{$v 3\left(F_{2}\right)$} & \multicolumn{2}{|c|}{$v 4\left(F_{2}\right)$} & \multicolumn{2}{|c|}{$v R\left(F_{1}\right)$} \\
\hline & $\mathrm{GeTe}_{4}$ & $\mathrm{SbTe}_{3}$ & $\mathrm{GeTe}_{4}$ & $\mathrm{SbTe}_{3}$ & $\mathrm{GeTe}_{4}$ & $\mathrm{SbTe}_{3}$ & $\mathrm{GeTe}_{4}$ & $\mathrm{SbTe}_{3}$ & $\mathrm{GeTe}_{4}$ & $\mathrm{SbTe}_{3}$ \\
\hline $\begin{array}{l}\mathrm{GeTe}_{4}[16] \\
\mathrm{SbTe}_{2}[16]\end{array}$ & 125 & 164 & 87 & 122 & 209 & 145 & 78 & 106 & 60 & 52 \\
\hline $\mathrm{Ge}_{15} \mathrm{Sb}_{15} \mathrm{Te}_{70}^{* *}$ & 127 & 157 & 92 & 127 & 214 & 142 & 80 & 109 & 65 & 56 \\
\hline $\mathrm{Ge}_{14} \mathrm{Sb}_{28} \mathrm{Te}_{56}^{* * *}$ & 117 & 164 & 91 & 134 & 214 & 146 & 79 & 91 & 66 & 55 \\
\hline
\end{tabular}

Примечание. ${ }^{*}-v 1$ и $v 3$ - валентные моды для симметричных и антисимметричных колебаний. $v 2$ и $v 4-$ деформационные моды симметричных и антисимметричных колебаний. $v R$ - внешняя мода для свободного вращения. ** — данные работы [15]. *** — данные настоящей работы.

пирамид $\mathrm{Sb}_{2} \mathrm{Te}_{3}$ [14]. Этот подход был развит в работе [15], в которой на основании расчета и сделанного ранее отнесения полос в спектрах $\mathrm{GeTe}_{4}[15]$ и $\mathrm{Sb}_{2} \mathrm{Te}_{3}[16]$ были отнесены полосы поглощения, обусловленные колебаниям на $\mathrm{Ge}-\mathrm{Te}$ и $\mathrm{Sb}-\mathrm{Te}$ связях в другом GST сплаве: $\mathrm{Ge}_{15} \mathrm{Sb}_{15} \mathrm{Te}_{70}$. В табл. 2 представлены данные по отнесению полос в фононном спектре $\mathrm{Ge}_{14} \mathrm{Sb}_{28} \mathrm{Te}_{56}$, полученные по той же схеме.

Кроме полос поглощения, обусловленных колебаниями на $\mathrm{Ge}-\mathrm{Te}$ и $\mathrm{Sb}-\mathrm{Te}$ связях, наиболее интенсивные из которых расположены на длинах волн 117 и $146 \mathrm{~cm}^{-1}$ соответственно, в фононном спектре $\mathrm{Ge}_{14} \mathrm{Sb}_{28} \mathrm{Te}_{56}$ присутствуют полосы, обусловленные колебаниями $\mathrm{Ge}-\mathrm{Ge}$ связей при $300 \mathrm{~cm}^{-1}$ [17] и $\mathrm{Te}-\mathrm{Te}$ связей при $168 \mathrm{~cm}^{-1}$ [18]. Слабые высокочастотные полосы при 228, 270 и $327 \mathrm{~cm}^{-1}$ могут быть отнесены к так называемому 2-фононному поглощению $2 v 1_{\mathrm{GeTe}}$, $v 1_{\mathrm{GeTe}}+v 3_{\mathrm{SbTe}}$ и $2 v 1_{\mathrm{SbTe}}$ соответственно.

Из сравнения спектральных параметров фононных спектров двух ХC сплавов системы GST: $\mathrm{Ge}_{15} \mathrm{Sb}_{15} \mathrm{Te}_{70}$ и $\mathrm{Ge}_{14} \mathrm{Sb}_{28} \mathrm{Te}_{56}$ видно, что положения полос поглощения и их интенсивности в этих спектрах существенно зависят от процентного содержания компонентов сплава. С удвоением количества $\mathrm{Sb}$, при увеличенном содержания Те в сплаве, окно прозрачности незначительно (на $\sim 10 \mathrm{~cm}^{-1}$ ), сдвигается в длинноволновую область ИК спектра, обеспечивая решение поставленной задачи.

\section{4. Заключение}

В работе проведено изучение оптических свойств халькогенидных сплавов на основе теллура, позволяющих работать в терагерцовом ИК диапазоне. С этой целью были синтезированы тройные сплавы систем $\mathrm{Ge}-\mathrm{Se}(\mathrm{Sb})-\mathrm{Te}$ и $\mathrm{Si}-\mathrm{Ge}(\mathrm{Ga})-\mathrm{Te}$, а также получены и проанализированы ИК спектры поглощения этих ХС в широком интервале длин волн от 0.75 до 300 мкм. Впервые особое внимание уделено фононным спектрам, так как именно они определяют длинноволновую границу окна прозрачности ХС. Идентификация полос поглощения по их положению и интенсивности, основанная на расчете и литературных данных, позволила установить, что из всех изученных ХС самым продвинутым в терагерцовую ИК область является сплав $\mathrm{Ge}_{14} \mathrm{Sb}_{28} \mathrm{Te}_{56}$.

Наиболее интенсивные полосы поглощения, обусловленные колебаниями на $\mathrm{Ge}-\mathrm{Te}$ и $\mathrm{Sb}-\mathrm{Te}$ связях в фононном спектре $\mathrm{Ge}_{14} \mathrm{Sb}_{28} \mathrm{Te}_{56}$, ограничивают длинноволновый край его прозрачности $\sim 35$ мкм. Считаем, что оптимизация $\mathrm{Ge}_{14} \mathrm{Sb}_{28} \mathrm{Te}_{56}$ по составу, очистка от примесей и термообработка будут способствовать дальнейшему снижению коэффициента поглощения этого сплава в длинноволновой ИК области спектра.

\section{Список литературы}

[1] G. Grischkowsky. Proc. 4th Int. Conf. on Infrared Phys. (Zurich, Switzerland, 1988) p. 51.

[2] H. Mantsch, D. Naumann. J. Molecul. Struct., 964 (1-3), 1 (2010).

[3] H. Martijn, A. Gromov, S. Smuk, H. Malm, C. Asplund, J. Borglind, S. Becanovic, J. Alverbro, U. Halldin, B. Hirschauer. Infrar. Phys. \& Technol., 47 (1-2), 106 (2006).

[4] D. Le Coq, C. Boussard-Plédel, G. Fonteneau, T. Pain, B. Bureau, J.-L. Adam. J. Non-Cryst. Sol., 326-327, 451 (2003).

[5] P. Lucas, Z. Yang, M. Fah, T. Lu, S. Jiang, C. Boussard-Pledel, M.-L. Anne, B. Bureauet. Optical Mater. Express, 3, 1049 (2013).

[6] V.B. Voloshinov, N. Gupta, L.A. Kulakova, V.S. Khorkin, B.T. Melekh, G.A. Knyazev. J. Opt., 18, 025402 (2016).

[7] Л.А. Кулакова, Б.Т. Мелех, С.А. Грудинкин, А.П. Данилов. ФТП. 47, 1435 (2013).

[8] S.A. Grudinkin, V.I. Bakharev, V.M. Egorov, V.T. Melekh, V.G. Golubev. Semiconductors, 45, 1462 (2011).

[9] A.R. Hilton, S. Kemp. Chalcogenide glass for infrared optics (N.Y.-Chicago-San Francisco-Lisbon, McGraw-Hill Companies, Inc., 2010).

[10] S. Cui, C. Boussard-Plédel, J. Lucas, B. Bureauet. Opt. Express, 22 (18), 21254 (2014).

[11] T. Kavetskyy, R. Golovchak, O. Shpotyukb, J. Filipeckic, J. Swiateket. Chalcogen. Letters, 1 (10), 125 (2004).

[12] D.K. Dwivedi, H.P. Pathak, R.K. Shukla. Amer. J. Mater. Sci. Engin., 1 (3), 46 (2013).

[13] V.F. Kokorina. Glasses for Infrared Optics (Boca RatonN.Y.-London-Tokyo, CRC Press, 1996). 
[14] J. Im, J. Eom, C. Park, K. Park, D-S. Suh, K. Kim, Y.-S. Kang, C. Kim, T.-Y. Lee, Y. Khang, Y.-G. Yoon, J. Ihmet. Phys. Rev. B 78, 205205 (2008).

[15] V.A. Ryzhov, D. Arsova. Phys. Sci. Int. J., 14 (4) 8 (2017).

[16] H. Ohkawa, M. Matsubara, N. Joshida. Patent US 5580632 A. Declared 17.08.1994. Published 03.11.1996. Bull. 1996/27.

[17] S. Kozyukhin, M. Veres, H.P. Nguyen, A. Ingram, V. Kudoyarova. Physics Procedia, 44, 82 (2013).

[18] K.S. Andrikopoulos, S.N. Yannopoulos, G.A. Voyiatzis, A.V. Kolobov, M. Ribes, J. Tominaga. J. Phys.: Condens. Mater., 18, 965 (2006).

Редактор А.Н. Смирнов

\section{Optical properties of chalcogenide tellurium based alloys in the far infrared region $(\lambda>30 \mu \mathrm{m})$}

V.A. Ryzhov , B.T. Melekh

loffe Institute,

194021 St. Petersburg, Russia

Abstract The ternary telluride alloys of systems $\mathrm{Ge}-\mathrm{Se}$ $(\mathrm{Sb})-\mathrm{Te}$ and $\mathrm{Si}-\mathrm{Ge}(\mathrm{Ga})-\mathrm{Te}$ were synthesized for to use at the terahertz frequencies. Are measured and investigated the transmission spectra obtained alloys over a wide wavelength range from 0.75 to 300 microns. The possible mechanisms of their formation are discussed. Comparative analysis of the results shows that the most promising is the alloy $\mathrm{Ge}_{14} \mathrm{Sb}_{28} \mathrm{Te}_{56}$ of the GST system, the phonon spectrum which lies in the range $40-280 \mathrm{~cm}^{-1}$, limiting long-wave alloy transparency window, near the thirty-five microns. Optimization of the composition $\mathrm{Ge}_{14} \mathrm{Sb}_{28} \mathrm{Te}_{56}$, removal of impurities and heat treatment will further reduce the absorption coefficient in the far-infrared spectrum of this alloy. 